\title{
EI BILINGÜISMO Y LA COMPRENSIÓN DE LECTURA EN ESTUDIANTES DE LA ESCUELA PROFESIONAL DE EDUCACIÓN SECUNDARIA EN LA UNIVERSIDAD NACIONAL DE SAN CRISTÓBAL DE HUAMANGA, 2016
}

\author{
ROJAS TELLO, Luis Lucio ${ }^{1}$ \\ ${ }^{1}$ Doctor en Ciencias de la Educación. Universidad Nacional de San Cristóbal de Huamanga. Ayacucho, \\ Perú
}

"El hombre es tantas veces hombre, cuanto más número de lenguas ha aprendido". Carlos I

\section{RESUMEN}

El objetivo del estudio es precisar la correlación que existe entre los niveles de bilingüismo castellano-quechua y los niveles de comprensión de lectora, en un el contexto educativo multilingüe y multicultural. La mayoría de los participantes de la muestra se ubican de acuerdo al uso y dominio de las lenguas en los niveles de bilingüismo: incipiente, subordinando y coordinado, en relación a los niveles de compresión de lectura: literal, inferencial y crítico valorativo. El estudio es cuantitativo, de nivel descriptivo correlacional transversal. Los instrumentos aplicados es la lista de cotejo para determinar el nivel dominio de dos lenguas y prueba de compresión de lectura para determinar el nivel de compresión en los estudiantes bilingües. Los resultados obtenidos evidencian que un porcentaje considerable de estudiantes obtuvieron notas aprobatorias, lo que indica que los promedios hallados en la compresión de lectura en términos generales se ubicado en el nivel cualitativo de "bueno". En conclusión, sí existe correlación positiva entre los niveles de bilingüismo y los niveles de la compresión de lectura. Por tanto, la condición de ser bilingüe no es obstáculo para el desarrollo cognitivo de los estudiantes universitarios, por el contrario, permite desarrollar mayores habilidades cognitivas en la aprehensión de dos culturas y ver la realidad de manera holística.

Palabras claves: Niveles de bilingüismo y niveles de comprensión de lectura

\section{SUMMARY}

The objective of the study is to determine the relationship between levels of bilingualism castellano-quechua and levels of reading comprehension, in a multilingual and multicultural educational context, one of the linguistic characteristics that predominate in the region of Ayacucho, most of the participants of the sample are located according to the use of language proficiency in the levels of bilingualism: incipient, subordinating and coordinated, in relation to the levels of reading comprehension: literal, inferential and critical value. The study is quantitative, descriptive cross-level correlational level. The applied instruments is the checklist to determine the proficiency level of two languages and reading compression test to determine the level of 
compression in bilingual students. The results obtained show that a considerable percentage of students obtained passing grades, which indicates that the averages found in reading comprehension in general terms are located in the qualitative level of "good". In conclusion, there is a positive correlation between levels of bilingualism and levels of reading comprehension. Therefore, the condition of being bilingual is not an obstacle to the cognitive development of university students, but on the contrary allows to develop greater cognitive skills in the apprehension of two cultures and to see reality in a holistic way.

Keywords: Levels of bilingualism and levels of reading comprehension

\section{INTRODUCCIÓN}

El estudio presenta uno de los temas actuales y transcendentales en el ámbito cultural y educativo en nuestro país. A pesar de tener una riqueza lingüista de cuarenta y siete lenguas con distintos grados de uso y dominio, no se aprovecha para ofrecer una educación de calidad de acuerdo al contexto sociolingüístico de Perú; consecuentemente, hasta la actualidad no hemos superado los estándares internacionales en la comprensión de lectura en la educación básica y superior, desencadenando una deficiente calidad educativa. El aprendizaje de nuevas culturas y lenguas son necesarias en nuestra sociedad megadiverso, y con mayor exigencia para nuestra región y país por sus características sociolingüísticas de ser multilingüe, pluricultural y multiétnica. La existencia de diferentes lenguas y culturas en nuestro país es una realidad innegable.

En el sistema educativo nacional, tradicionalmente ha primado la enseñanza de la lengua española, a pesar de la necesidad de revalorar y consolidar la lengua materna para fortalecer la identidad lingüística y cultural. Consideramos impostergable enseñanza nuevas lenguas y culturas en la sociedad actual, para evitar los conflictos innecesarios habitualmente originados por la incomprensión y la desconfianza hacia lo desconocido.

El bilingüismo ha sido estudiado ampliamente, por Albó (1974), Appely Muysken (1996) Baker (1997), Escobar (2000), Julca (2000). Para nosotros el bilingüismo, tiene un conocimiento más equilibrado de dos lenguas; mientras la persona que aprende una segunda lengua presenta un mayor dominio de la lengua materna (L1) que de segunda lengua (L2). La lengua siempre se utilizado dentro de un determinado contexto sociocultural, que varía de una lengua a otra: una persona que conozca dos lenguas es más bicultural 0 multicultural. Esta relación es inherente a la competencia o dominio que tenga un individuo de dos o más lenguas.

En cuanto a la comprensión de lectura se consideró los planteamientos de Cassany (2009), Aliaga (2012), Lugo (2007), Pinzas (2006), Ruíz (2011), Solé (2000), etc. Consideramos esta actividad como uno de los pilares fundamentales para la formación académica de los estudiantes universitarios en la adquisición de conocimientos en su proceso formación profesional. Los expertos ya citados, consideran la lectura como un acto complejo cuya construcción necesita de saberes previos, pues tiene sus objetivos, características, valores y uso de técnicas.

El estudio del bilingüismo y la compresión de lectura se basan en la preocupación incesante de esclarecer dudas planteados de las personas bilingües, porque es frecuente escuchar que, ser bilingües repercute negativamente en el desarrollo cognitivo; por tanto, los resultados obtenidos aseveran categóricamente, que el bilingüismo y la compresión lectora tiene una correlación positiva considerable, con igual promedio de logros; es decir, los estudiantes que se ubican en el nivel de bilingüismo subordinado y coordinado logran resultados positivos en la compresión literal, inferencial y crítica; sin embargo, los bilingües incipientes no logran los resultados deseados 
en la compresión de lectura. Los estudiantes bilingües dentro del escenario universitario se encuentran entre dos culturas: la andina y la occidental. Cada una de ellas presenta diversas particularidades en su modo de vida y en la forma de concebir el mundo. El currículo de estudio vigente en el sistema universitario contiene exiguas áreas para desarrollar la educación intercultural bilingüe de calidad; es así, que en algunas escuelas de formación profesional, se desarrollan los cursos de quechua y EIB; para promover la compresión lectora en lengua quechua y conocer la cultura andina.
El enfoque de la investigación es cuantitativo no experimental, con dos variables de estudio: variable $A$, niveles bilingüismo; variable $B$, niveles de comprensión de lectura. Tipo de investigación descriptiva correlacional transversal. La técnica de recopilación de datos fue a través de la observación y examen; para el primer caso, se aplicó como instrumento la lista de cotejo, para determinar el nivel de bilingüismo: incipiente, subordinado y coordinado; asimismo, para el segundo caso, se aplicó la prueba de compresión de lectura para precisar el nivel: literal, inferencial y crítico valorativo. Para la validez y confiabilidad de los instrumentos fueron sometidos a juicio de expertos. Finalmente se aplicó el coeficiente de confiabilidad de alfa de Cronbach que fueron superiores a $82 \%$.

\section{Métodosy materiales Resultados}

\section{Correlación entre las variables: A y B}

\section{Tabla № 1 Matriz de correlación de Pearson del bilingüismo y los niveles de compresión de lectura}

\begin{tabular}{|c|c|c|c|c|c|c|}
\hline & & BILINGUISMO & $\begin{array}{l}\text { BILINGUISMO } \\
\text { CUALITATIVO }\end{array}$ & $\begin{array}{l}\text { NIVEL } \\
\text { LITERAL }\end{array}$ & $\begin{array}{l}\text { NIVELL } \\
\text { INFEREENCIA } \\
\text { LER }\end{array}$ & $\begin{array}{l}\text { NIVEL } \\
\text { CRITICO }\end{array}$ \\
\hline BILINGUISMO & $\begin{array}{l}\text { Pearson Correlation } \\
\text { Sig. (2-tailed) } \\
\mathrm{N}\end{array}$ & 5 & $\begin{array}{r}884^{\prime \prime} \\
, 000 \\
50\end{array}$ & $\begin{array}{r}.474^{-\prime} \\
, 001 \\
50\end{array}$ & $\begin{array}{r}.480^{-\prime} \\
.000 \\
50\end{array}$ & $\begin{array}{r}, 553^{-1} \\
, 000 \\
50\end{array}$ \\
\hline $\begin{array}{l}\text { BILINGUISMO EN } \\
\text { CUALITATIVO }\end{array}$ & $\begin{array}{l}\text { Pearson Correlation } \\
\text { Sig. (2-tailed) } \\
\text { N }\end{array}$ & $\begin{array}{r}884^{-} \\
, 000 \\
50\end{array}$ & $\begin{array}{l}1 \\
50\end{array}$ & $\begin{array}{r}, 346^{*} \\
, 014 \\
50\end{array}$ & $\begin{array}{r}.525 \\
.000 \\
50\end{array}$ & $\begin{array}{r}, 618^{-} \\
.000 \\
50\end{array}$ \\
\hline NIVEL LITERAL & $\begin{array}{l}\text { Pearson Correlation } \\
\text { Sig. (2-tailed) } \\
\text { N }\end{array}$ & $\begin{array}{r}4,474 \\
, 001 \\
50\end{array}$ & $\begin{array}{r}, 346^{\circ} \\
, 014 \\
50\end{array}$ & 1 & $\begin{array}{r}.342^{-} \\
.015 \\
50\end{array}$ & $\begin{array}{r}.275 \\
.053 \\
50\end{array}$ \\
\hline NIVEL INFERENCIAL & $\begin{array}{l}\text { Pearson Correlation } \\
\text { Sig. (2-tailed) } \\
\text { N }\end{array}$ & $\begin{array}{r}480^{-\prime} \\
.000 \\
50\end{array}$ & $\begin{array}{r}, 525 \% \\
, 000 \\
50\end{array}$ & $\begin{array}{r}, 342^{*} \\
, 015 \\
50\end{array}$ & $\begin{array}{l}1 \\
50\end{array}$ & $\begin{array}{r}, 366^{-1} \\
, 009 \\
50\end{array}$ \\
\hline NIVEL CRITICO & $\begin{array}{l}\text { Pearson Correlation } \\
\text { Sig. (2-tailed) } \\
\mathrm{N}\end{array}$ & $\begin{array}{r}.553^{-"} \\
.000 \\
50\end{array}$ & $\begin{array}{r}618^{*-} \\
, 000 \\
50\end{array}$ & $\begin{array}{r}, 275 \\
, 053 \\
50\end{array}$ & $\begin{array}{r}.366^{-\prime} \\
, 009 \\
50\end{array}$ & $\begin{array}{l}1 \\
50\end{array}$ \\
\hline
\end{tabular}

$\because$ Correlation is significant at the 0.01 level (2-tailed).

Es evidente, la correlación de Pearson calculadas en base a las calificaciones del bilingüismo y de comprensión de lectura, expresadas en escala vigesimal; la prueba de Correlación de Pearson se aplicó en el Software SPSS con $95 \%$ de confianza y $5 \%$ de significancia denotada en el cuerpo de la tabla con $\left(^{*}\right)$, y con $99 \%$ de confianza y $1 \%$ de significancia denotado con $\left({ }^{* *}\right)$, en el primer caso, se interpreta como una correlación significativa y en el segundo caso altamente significativa. En la interpretación de estos estadígrafos presentados en las relaciones de interés, se combina con la escala de correlación tradicional mencionada líneas arriba. En consecuencia, la correlación 
entre "el bilingüismo y el nivel literal de la comprensión de lectura" es moderada, dado que tiene un estadígrafo $r=0,474^{* *}$, la relación entre "el bilingüismo y el nivel inferencial de la comprensión de lectura" es moderada con un $r=0,480^{* *}$ y la relación entre "el bilingüismo y el nivel crítico de la comprensión de lectura" también es moderada con un $r=0,553^{* *}$. Los resultados de las correlaciones de Pearson entre el bilingüismo y los niveles de comprensión de lectura, indican que hay una relación positiva entre las calificaciones obtenidas en el

Tabla $\mathbf{N}^{\circ} 2$ Matriz de correlación de Sperman del bilingüismo y los niveles de compresión de lectura

\begin{tabular}{|c|c|c|c|c|c|c|c|}
\hline & & & BILINGUISMO & $\begin{array}{l}\text { BILINGUISMO } \\
\text { CUALITATIVO }\end{array}$ & $\begin{array}{l}\text { NIMEL } \\
\text { LITERAL }\end{array}$ & $\begin{array}{l}\text { NIVELL } \\
\text { INFERENCIA }\end{array}$ & $\begin{array}{l}\text { NIVEL } \\
\text { CRITICO }\end{array}$ \\
\hline \multirow[t]{5}{*}{ Spearman's rho } & BILINGUISMO & $\begin{array}{l}\text { Correlation Coefficient } \\
\text { Sig. (2-tailed) } \\
\mathrm{N}\end{array}$ & $\begin{array}{r}1,000 \\
50 \\
\end{array}$ & $\begin{array}{r}930^{\circ} \\
.000 \\
50 \\
\end{array}$ & $\begin{array}{r}.385^{\circ} \\
.006 \\
50 \\
\end{array}$ & $\begin{array}{r}.368^{-"} \\
.009 \\
50\end{array}$ & $\begin{array}{r}.666^{\prime \prime} \\
.000 \\
50\end{array}$ \\
\hline & $\begin{array}{l}\text { BILINGUISMO EN } \\
\text { CUALITATIVO }\end{array}$ & $\begin{array}{l}\text { Correlation Coefficient } \\
\text { Sig. (2-tailed) } \\
\mathrm{N}\end{array}$ & $\begin{array}{r}.930^{\circ} \\
.000 \\
50\end{array}$ & $\begin{array}{r}1,000 \\
50\end{array}$ & $\begin{array}{r}.253 \\
.076 \\
50\end{array}$ & $\begin{array}{r}.431^{\prime \prime} \\
.002 \\
50\end{array}$ & $\begin{array}{r}.638^{\circ} \\
.000 \\
50\end{array}$ \\
\hline & NIVEL LITERAL & $\begin{array}{l}\text { Correlation Coefficient } \\
\text { Sig. (2-tailed) } \\
\mathrm{N}\end{array}$ & $\begin{array}{r}.385^{*} \\
.006 \\
50\end{array}$ & $\begin{array}{r}.253 \\
.076 \\
50\end{array}$ & $\begin{array}{r}1,000 \\
50\end{array}$ & $\begin{array}{r}.111 \\
.442 \\
50\end{array}$ & $\begin{array}{r}.298^{\circ} \\
.035 \\
50\end{array}$ \\
\hline & NIVEL INFERENCIAL & $\begin{array}{l}\text { Correlation Coefficient } \\
\text { Sig. (2-tailed) } \\
\mathrm{N}\end{array}$ & $\begin{array}{r}.368^{*} \\
.009 \\
50\end{array}$ & $\begin{array}{r}.431^{\circ} \\
.002 \\
50\end{array}$ & $\begin{array}{r}.111 \\
.442 \\
50\end{array}$ & $\begin{array}{r}1,000 \\
50\end{array}$ & $\begin{array}{r}.283^{\circ} \\
.046 \\
50\end{array}$ \\
\hline & NIVEL CRITICO & $\begin{array}{l}\text { Correlation Coefficient } \\
\text { Sig. (2-tailed) } \\
\mathrm{N}\end{array}$ & $\begin{array}{r}.666^{*} \\
.000 \\
50\end{array}$ & $\begin{array}{r}.638^{\circ} \\
.000 \\
50\end{array}$ & $\begin{array}{r}.298^{\circ} \\
.035 \\
50\end{array}$ & $\begin{array}{r}.283^{\circ} \\
.046 \\
50\end{array}$ & $\begin{array}{r}1,000 \\
50\end{array}$ \\
\hline
\end{tabular}

$\because$ Correlation is significant at the 0.01 level (2-tailed).

Las correlación de Spearman aplicadas a las calificaciones del bilingüismo y comprensión de lectura convertidas a la escala ordinal, cuyos resultados presentan un coeficiente de correlación $r_{s}=0.385^{\star *}$ para la relación que existe entre "el bilingüismo y el nivel literal de la comprensión de lectura", indica que entre estas variables hay una relación baja; para la relación entre "el bilingüismo y el nivel inferencial de la comprensión de lectura" presenta un $r_{\mathrm{s}}=0.368^{* *}$ indica también una relación baja y para la asociación de "el bilingüismo y el nivel crítico de la comprensión de lectura" con un $r=0.666^{* *}$ indica una relación alta. Comparando los resultados de la correlación de Pearson

bilingüismo son directamente proporcionales a las calificaciones obtenidas en los niveles de comprensión lectora, que asociando a los promedios y desviaciones descritas en los párrafos anteriores, se determina que hay estabilidad en las calificaciones, con mayor incidencia en el bilingüismo subordinado y bueno para la comprensión de lectura en sus tres niveles.

\section{Prueba de hipótesis}


Tabla 3 Distribución bidimensional de frecuencias del bilingüismo y el nivel literal de compresión de lectura

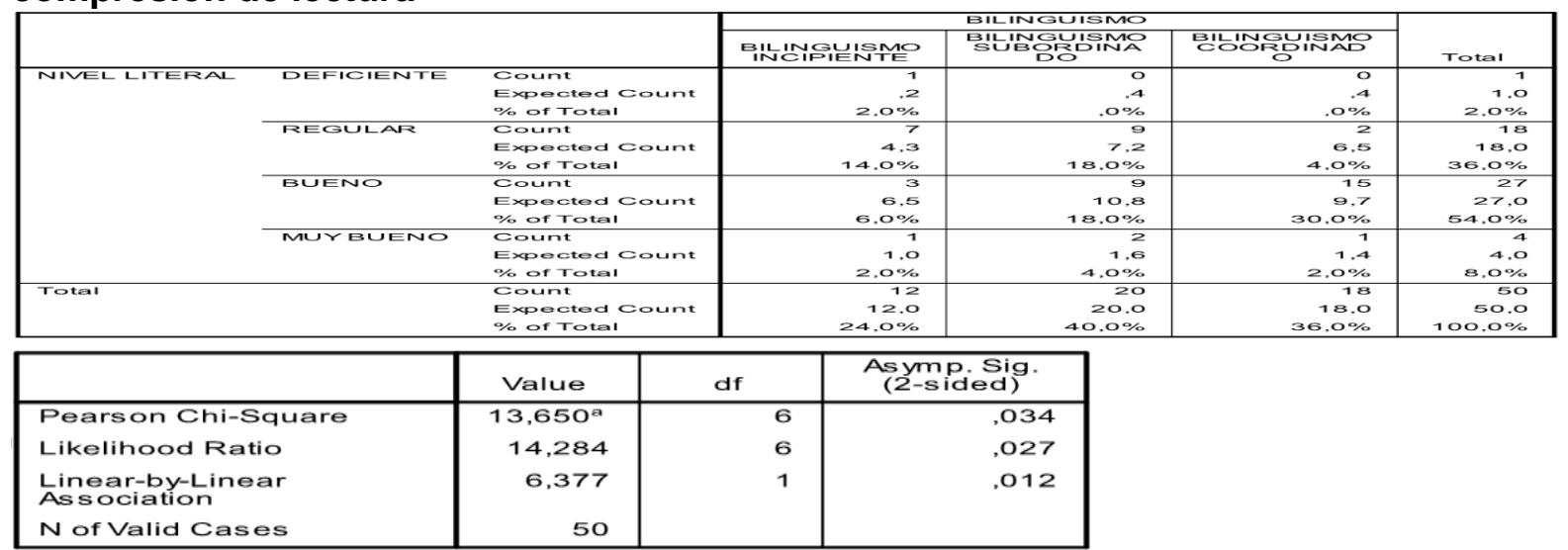

a. 7 cells $(58.3 \%)$ have expected count less than 5 . The

En la tabla 3, sabemos que si las diferencias entre las frecuencias observadas y las esperadas son pequeñas formaran un estadístico Chi-Cuadrado pequeño; por consiguiente, se dará consistencia a la hipótesis nula de esta prueba, caso contrario, si las diferencias son grandes se formará un valor Chi-Cuadrado grande, que proporcionará consistencia a la hipótesis alterna; así mismo, si aplicamos el criterio de que las categorías que tengan frecuencias esperadas menores o iguales a 5 , deben ser reagrupadas a la categoría inmediata superior, de tal forma que mejoría el valor de la prueba Chi-Cuadrado. Con estos dos aspectos descritos en el párrafo anterior se eliminaría de la tabla 3 , las categorías de bilingüismo incipiente, el nivel literal de calificativo "deficiente" y "muy bueno", mejorando las frecuencias de las otras categorías próximas de fila o de columna; pero, si analizamos tal como se muestra en la tabla, se determina que las diferencias que hay entre las frecuencias conjuntas observadas y las esperadas para la categoría del bilingüismo incipiente asociada a la categoría del nivel literal, se determina que las diferencias son pequeñas y que no ayudan a la formación del estadígrafo ChiCuadrado y por consiguiente tampoco a la formación del índice de correlación de Spearman.

\section{Prueba hipótesis específicas}

$\mathbf{H}_{0}$ : Las calificaciones obtenidas entre el bilingüismo y el nivel literal de comprensión de lectura son independientes.

$\mathbf{H}_{\mathbf{a}}$ : Las calificaciones obtenidas entre el bilingüismo y el nivel literal de comprensión de lectura están relacionadas.

Los resultados del test Chi-Cuadrado, evaluados con el $95 \%$ de confianza y el $5 \%$ de significancia, presentan un estadígrafo $\mathbb{1}^{2}$ $=13,650$ con 6 grados de libertad y un $p-$ Valor igual a 0,034 que es menor que el nivel de significancia $\alpha=0,05$, esto significa que la muestra aporta información suficiente para rechazar la hipótesis nula y por consiguiente se determina que las calificaciones obtenidas entre el bilingüismo y el nivel literal de comprensión de lectura están relacionadas. Estos resultados obtenidos a través del test Chi-Cuadrado confirman los resultados obtenidos en las correlaciones de Pearson y Spearman. En consecuencia, se tiene elementos suficientes para afirmar que el bilingüismo está relacionado con el nivel 
literal de la capacidad de comprensión de lectura. Por tanto, se puede determinar que:

- El bilingüismo incipiente no está relacionado con las categorías del nivel literal de la capacidad de comprensión de lectura, dado que las diferencias entre las frecuencias bidimensionales observadas $y$ esperadas son pequeñas, y no aportan mucho a la formación del estadígrafo Chi-Cuadrado

- El bilingüismo subordinado está relacionado con las categorías del nivel literal de la capacidad de comprensión de lectura, dado que las diferencias entre las frecuencias bidimensionales observadas y esperadas son grandes, para los cruces "bilingüismo subordinado y nivel literal de calificación "regular" y "bilingüismo subordinado y nivel literal de calificación "bueno" que ayudan a la formación del estadígrafo ChiCuadrado y en los otros cálculos de correlación indican que hay proporcionalidad directa entre los cruces de dichas categorías; asimismo, es evidente que los calificativos están contenidos en un intervalo de notas de $[11,16]$.

- El bilingüismo coordinado está relacionado con las categorías del nivel literal de la capacidad de comprensión de lectura, pues la diferencia entre las frecuencias bidimensionales observadas y esperada es grande, cuyo mayor aporte lo proporciona el cruce de las categorías "bilingüismo coordinado y nivel literal bueno"

Tabla 4. Distribución bidimensional de frecuencias del bilingüismo y el nivel inferencial de compresión de lectura

\begin{tabular}{|c|c|c|c|c|c|c|}
\hline & & & \multicolumn{3}{|c|}{ BILINGUISMO } & \multirow[b]{2}{*}{ Total } \\
\hline & & & $\begin{array}{l}\text { BILINGUISMO } \\
\text { INCIPIENTE }\end{array}$ & $\begin{array}{l}\text { BILINGUISMO } \\
\text { SUBORDINA } \\
\text { DO }\end{array}$ & $\begin{array}{l}\text { BILINGUISMO } \\
\text { COORDINAD } \\
\text { O }\end{array}$ & \\
\hline \multirow[t]{9}{*}{ NIVEL INFERENCIAL } & REGULAR & Count & 1 & 4 & $\bar{O}$ & 5 \\
\hline & & Expected Count & 1,2 & 2,0 & 1,8 & 5,0 \\
\hline & & $\%$ of Total & $2,0 \%$ & $8,0 \%$ &, $0 \%$ & $10,0 \%$ \\
\hline & BUENO & Count & 11 & 16 & 13 & 40 \\
\hline & & Expected Count & 9,6 & 16,0 & 14,4 & 40,0 \\
\hline & & $\%$ of Total & $22,0 \%$ & $32,0 \%$ & $26,0 \%$ & $80,0 \%$ \\
\hline & MUYBUENO & Count & o & o & 5 & 5 \\
\hline & & Expected Count & 1,2 & 2,0 & 1,8 & 5,0 \\
\hline & & $\%$ of Total &, $0 \%$ & , $0 \%$ & $10,0 \%$ & $10,0 \%$ \\
\hline \multirow[t]{3}{*}{ Total } & & Count & 12 & 20 & 18 & 50 \\
\hline & & Expected Count & 12,0 & 20,0 & 18,0 & 50,0 \\
\hline & & $\%$ of Total & $24,0 \%$ & $40,0 \%$ & $36,0 \%$ & $100,0 \%$ \\
\hline
\end{tabular}

\begin{tabular}{|l|r|r|r|}
\hline & \multicolumn{1}{|c|}{ Value } & df & \multicolumn{1}{c|}{$\begin{array}{c}\text { Asymp. Sig. } \\
\text { (2-sided) }\end{array}$} \\
\hline Pearson Chi-Square & $13,063^{\mathrm{a}}$ & 4 &, 011 \\
Likelihood Ratio & 15,733 & 4 &, 003 \\
Linear-by-Linear & 6,025 & 1 &, 014 \\
Ass Ociation & 50 & & \\
N of Valid Cases & & & \\
\hline
\end{tabular}

a. 6 cells $(66.7 \%)$ have expected count less than 5 . The

Fuente: Guía de observación para ubicar los niveles de bilingüismo y prueba de comprensión de lectura para el nivel inferencial

Los resultados del estadígrafo Chi-Cuadrado para la siguiente hipótesis:

$\mathrm{H}_{0}$ : Las calificaciones obtenidas entre el bilingüismo y el nivel inferencial de comprensión lectora son independientes.
$\mathbf{H}_{\mathbf{a}}$ : Las calificaciones obtenidas entre el bilingüismo y el nivel inferencial de comprensión de lectura están relacionadas.

Los resultados del Test Chi-Cuadrado, evaluados con el $95 \%$ de confianza y el $5 \%$ de significancia, presentan un estadígrafo $\mathbb{\Downarrow}^{2}=13,063$ con 4 
grados de libertad y un $p-$ Valor igual a 0,011 que es menor que el nivel de significancia $\alpha=$ 0,05, esto significa que la muestra aporta información suficiente para rechazar la hipótesis nula y por consiguiente se precisa que las calificaciones obtenidas entre el bilingüismo y el nivel inferencial de comprensión de lectura están relacionadas.
Estos resultados obtenidos en el Test ChiCuadrado, en las correlaciones de Pearson y de Spearman, permiten precisar que los mayores aportes para formación de estos estadígrafos están en la asociación de las tres categorías del bilingüismo y el nivel inferencial de calificativo de "Bueno" y en los demás casos aportan poco.

Tabla $N^{\circ} 5$. Distribución bidimensional de frecuencias del bilingüismo y el nivel crítico de compresión de lectura

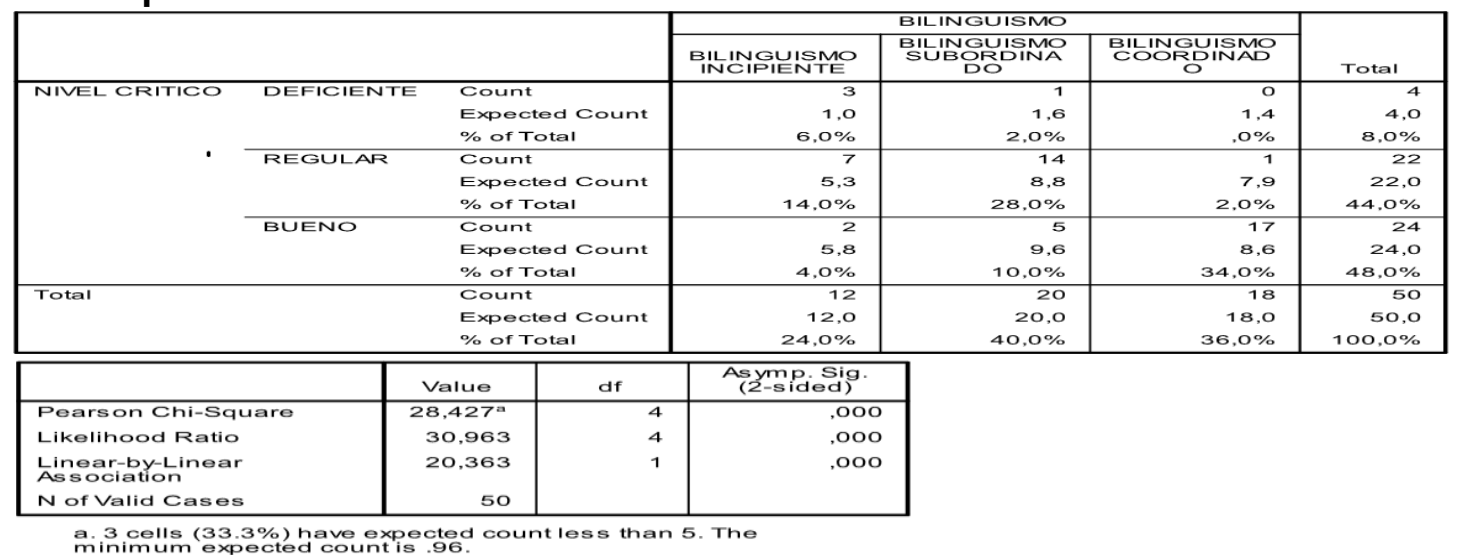

Fuente: Guía de observación para ubicar los niveles de bilingüismo y prueba de comprensión de lectura para el nivel crítico

En la tabla 5 , se tiene que el $28 \%$ de los estudiantes presentan bilingüismo subordinado y nivel crítico de calificativo "regular", el $34 \%$ muestran un bilingüismo coordinado y un nivel crítico de calificativo "bueno", en los demás casos se observan porcentajes bajos. En la tabla adjunta, se tiene los resultados del estadígrafo ChiCuadrado para el siguiente para de hipótesis:

$\mathbf{H}_{0}$ : Las calificaciones obtenidas entre el bilingüismo y el nivel crítico de comprensión de lectura son independientes.

$\mathbf{H}_{\mathbf{a}}$ : Las calificaciones obtenidas entre el bilingüismo y el nivel crítico de comprensión de lectura están relacionadas.

Los resultados del Test Chi-Cuadrado, evaluados con el $95 \%$ de confianza y el $5 \%$ de significancia, presentan un estadígrafo $\mathbb{1}^{2}$ $=28,427$ con 4 grados de libertad y un $p-$
Valor igual a 0,000 que es menor que el nivel de significancia $\alpha=0,05$, esto significa que la muestra aporta información suficiente para rechazar la hipótesis nula y por consiguiente se determina que las calificaciones obtenidas entre el bilingüismo y el nivel inferencial de comprensión de lectura están relacionadas. Haciendo el análisis conjunto de los resultados obtenidos en el Test Chi Cuadrado, en las correlaciones de Pearson y de Spearman para esta parte, permiten determinar que los mayores aportes para formación de estos estadígrafos está en la asociación de las categorías del bilingüismo subordinado con el nivel crítico de calificativo "regular" y bilingüismo coordinado con el nivel crítico de calificativo "bueno" y en los demás casos aportan poco.

Evaluando los resultados en forma conjunta, se precisa que la capacidad de comprensión de lectura para sus tres niveles en la escala 
de calificación cualitativa es "regular" o "bueno" y está latente el bilingüismo subordinado o coordinado, por otro lado, se puede afirmar que hay poca presencia de calificativos "deficientes" o "muy buenos" en la capacidad de comprensión de lectura. En nivel crítico de comprensión lectora se observa que les cuesta mayor trabajo porque son pocos los estudiantes que hay logrado el calificativo de "muy bueno".

\section{Discusión}

Correlaciones de Pearson registrados presentados en los resultados, la correlación del bilingüismo con el nivel literal de comprensión lectora es baja con $r=0,346^{*}$, el bilingüismo con el nivel inferencial de comprensión de lectura es moderada con $r=$ $0,525^{\star \star}$ y el bilingüismo con el nivel crítico de comprensión de lectura presenta una relación alta con $r=0,618^{* *}$. Estas asociaciones diferencian los grados de relación que existe entre el bilingüismo y la comprensión de lectura; con los cálculos posteriores se identifica otros patrones de comportamiento de las variables en análisis.

La correlaciones de Spearman aplicadas a las calificaciones del bilingüismo $y$ comprensión de lectura convertidas a la escala ordinal, cuyos resultados presentan un coeficiente de correlación $r_{s}=0,385^{\star \star}$ para la relación que existe entre "el bilingüismo y el nivel literal de la comprensión de lectura", lo que indica que entre estas variables hay una relación baja; para la relación entre "el bilingüismo y el nivel inferencial de la comprensión de lectura" presenta un $r_{s}=0,368^{* *}$ que indica también una relación baja y para la asociación de "el bilingüismo y el nivel crítico de la comprensión de lectura" con un $r=0,666^{* *}$ que indica una relación alta.
Comparando los resultados hallados en la correlación de Pearson y la correlación de Spearman, se establece que existe correlación entre el bilingüismo y la comprensión de lectura. En el primer cálculo los resultados son más optimistas que el segundo; pero, apertura la posibilidad que aplicar la prueba de Tets Chi-Cuadrado, para el bilingüismo y el nivel literal de comprensión de lectura; por consiguiente, se establece que las calificaciones obtenidas entre el bilingüismo y el nivel literal de comprensión de lectura están relacionadas

Estos resultados obtenidos a través del Test Chi-Cuadrado, confirman los resultados obtenidos en las correlaciones de Pearson y Spearman. El bilingüismo incipiente no está relacionado con las categorías del nivel literal de la capacidad de comprensión de lectura, dado que las diferencias entre las frecuencias bidimensionales observadas $y$ esperadas son pequeñas, y no aportan mucho a la formación del estadígrafo ChiCuadrado. Asimismo, se tiene elementos suficientes para afirmar que el bilingüismo está relacionado con el nivel inferencial de la comprensión de lectura.

El bilingüismo subordinado, está relacionado con las categorías del nivel literal de la capacidad de comprensión de lectura, dado que las diferencias entre las frecuencias bidimensionales observadas y esperadas son grandes, para los cruces "bilingüismo subordinado y nivel literal es regular" $y$ "bilingüismo subordinado y nivel literal es bueno" que ayudan a la formación del estadígrafo Chi-Cuadrado y en los otros cálculos de correlación indican que hay proporcionalidad directa entre los cruces de dichas categorías; además, es evidente que los calificativos están contenidos en un intervalo de $[11,16]$.

El bilingüismo coordinado, está relacionado con las categorías del nivel literal de la 
capacidad de comprensión de lectura, pues la diferencia entre las frecuencias bidimensionales observadas y esperada es grande, cuyo mayor aporte proporciona el cruce de las categorías "bilingüismo coordinado y nivel literal de calificativo "bueno".

Las calificaciones obtenidas entre el bilingüismo $y$ el nivel inferencial de comprensión lectora están relacionadas. Realizando el análisis conjunto de los resultados obtenidos en el Test ChiCuadrado, en las correlaciones de Pearson y de Spearman, permiten determinar que los mayores aportes para formación de estos estadígrafos está en la asociación de las categorías del bilingüismo subordinado con el nivel crítico "regular" y bilingüismo coordinado con el nivel crítico "bueno".

Evaluando la información en forma conjunta, se determina que en la capacidad de comprensión lectora para los tres niveles en la calificación de la escala es "regular y bueno" y está latente el bilingüismo subordinado o coordinado; por otro lado, se puede afirmar que hay poca presencia de calificativos "deficientes" o "muy bueno" en la capacidad de comprensión de lectura. En nivel crítico, se observa que demanda mayor trabajo porque son pocos los estudiantes que han logrado el calificativo de "muy bueno".

En contraste con otras investigaciones coincidimos en los resultados de Morales (2009) Bilingüismo y el rendimiento académico de estudiantes de nivel secundaria de la instituciones educativas de la región de Ayacucho: concluye, que el bilingüismo en la región de Ayacucho no influye negativamente en el rendimiento académico; por el contrario, debe ser una cualidad lingüística que permita un mejor desenvolvimiento académico. Los estudiantes de la zona urbano marginal nos son influidos negativamente en su rendimiento académicos su característica de ser bilingüe.

Cabanillas (2004) en la investigación "Influencia de la enseñanza directa en el mejoramiento de la comprensión lectora de los estudiantes de la Facultad de Ciencias de la Educación de la Universidad Nacional San Cristóbal de Huamanga", concluye: "que la estrategia de enseñanza directa ha mejorado significativamente (tanto estadística como pedagógico-didáctica) la comprensión lectora en dichos estudiantes".

Del mismo modo, Padrón (2001) realizó la investigación "La influencia de estrategias de aprendizaje en la comprensión lectora", aplicando tres instrumentos para evaluar los resultados. Las conclusiones indican que los estudiantes de inglés que aplican estrategias metacognitivas de aprendizaje tienen mejores niveles académicos de comprensión lectora, que aquellos que no utilizan este tipo de estrategias. Los resultados obtenidos demuestran, que el bilingüismo tiene ventajas en el desenvolvimiento sociocultural, académico, etc. con mayor énfasis en la compresión de lectura, por lo que valora de la siguiente manera.

La competencia comunicativa es transcendental en un contexto sociolingüístico, multilingüe y pluricultural, para construir un país inclusivo en la educación como un derecho. En Ayacucho en mayor porcentaje, los estudiantes son de la zona rural, por ende, son bilingües, por lo que requiere profesionales con perfil bilingüe, para responder a las necesidades educativas de los estudiantes bilingües. Además, la creación de nuevas instituciones EIB en las zonas rurales y urbano-marginales para acceder a la educación de calidad e inclusiva.

Está demostrado que, el bilingüismo favorece a la comprensión de lectura, la tolerancia 
entre los individuos y los pueblos, porque cada uno conoce sus propias costumbres, tradiciones y forma de vida, etc. por todo ello el Perú es eminentemente megadiverso. De la misma manera, el bilingüismo favorece una flexibilidad mental, porque el individuo conoce dos culturas con sus características particulares y sabe desenvolverse en cada una de ellas, en comparación con un individuo monolingüe. Además, el bilingüismo enriquece la personalidad del hombre gracias al conocimiento de dos culturas.

Coincidimos con Arnberg (1993): el bilingüismo es importante para la sociedad; el bilingüismo ofrece ventajas a nivel individual, brinda oportunidades más amplias de las que posee la persona monolingüe y monocultural, tales como la oportunidad de vivir y trabajar en otra nación. Muchos padres mencionan los efectos estimulantes del bilingüismo por la capacidad de razonamiento de sus hijos, por el acceso a las ideas y experiencias más amplias, y una experiencia que significa acercarse a dos culturas, algo que no ocurre en un niño monolingüe. Así mismo, Baker (1996: 11-13) refiere sobre las ventajas de ser bilingüe influye sobre la identidad del individuo, las relaciones sociales, la enseñanza, la ocupación, residencia, los viajes y la manera de pensar. Ser bilingüe no solamente significa saber hablar dos idiomas, sino, esta habilidad tiene consecuencias pedagógicas, sociales, económicas, culturales y políticas.

\section{Conclusiones}

Sí existe correlación positiva entre los niveles de bilingüismo y los niveles de la compresión de lectura, en tal sentido, el bilingüismo no tiene consecuencias negativas en el desarrollo cognitivo de los estudiantes en cualquier nivel o modalidad de la educación peruana, tal como se demuestra en los resultados.
No existe relación positiva entre el bilingüismo incipiente castellano-quechua $y$ los niveles de compresión literal, inferencial y crítico en los estudiantes, puesto que los resultados determinan que diferencias entre las frecuencias bidimensionales observadas y esperadas son pequeñas, y no aportan mucho a la formación del estadígrafo ChiCuadrado estadígrafo $\mathbb{\mathbb { I }}^{2}=13,650$ con 6 grados de libertad y un $p$ - Valor igual a 0,034 .

Sí existe una relación positiva entre el bilingüismo subordinado castellano-quechua y los niveles de compresión literal, inferencial y crítico, se observa que el bilingüismo subordinado combinado con las capacidades de la comprensión de lectura ayuda a la formación de los Estadígrafos Chi Cuadrado. $\mathbb{I}^{2}=13.650$ con un $p-$ valor de $0.034, \mathbb{1}^{2}$ $=13.063$ con un $p-$ valor de 0.011 y $\mathbb{1}^{2}$ $=28.427$ con un $p-$ valor de 0.000 .

Sí existe una relación positiva entre el bilingüismo coordinado castellano-quechua y los niveles de compresión literal, inferencial y crítico, se observa que el bilingüismo coordinado combinado con los niveles de la comprensión de lectura ayuda a la formación de los Estadígrafos Chi Cuadrado. $\mathbb{1}^{2}$ $=13,650$ con un $p-$ valor de 0,034, $\mathbb{I}^{2}$ $=13,063$ con un $p-$ valor de 0,011 y $\mathbb{1}^{2}$ $=28,427$ con un $p-$ valor de 0,000 .

Correspondencia

Luis Lucio Rojas Tello

Av. Ramón Castilla № 811 Huamanga-Ayacucho Correo: unsch2004@hotmail.com

Teléfono 990-860006

\section{Referencias bibliográficas}

Aliaga, L. (2012)Comprensión lectora y rendimiento académico en comunicación de alumnos del Segundo Grado de una Institución Educativa de Ventanilla. Lima-Perú 
Appel, R. y Muysken, P. (1996). Bilingüismo y contacto de lenguas. Barcelona: Ariel.

Baker, C. (1997). Fundamentos de educación bilingüe y bilingüismo. Madrid: Cátedra, S.A.

Cabanillas, G. (2013). Cómo hacer tesis en educación y ciencias afines. Perú: Educación.

Callo, D. (2015). "Interferencia gramatical en el quechua de hablantes bilingües del valle de colca". (1ra ed.). Lima, Perú.

Cassany, D. (2009). Enseñar lengua. Barcelona: Graó

Colque, M y Bohórquez, J. (2006) Logros $y$ dificultades en el desarrollo de la comprensión lectora y producción de textos narrativos en segunda lengua. Cochabamba-Bolivia

Cummins, J. (2002). Lenguaje, poder y pedagogía. Madrid: Morata.

Escobar, A. M. (2000). Contacto social y lingüístico. Lima: Fondo Editorial de la Pontificia Universidad Católica del Perú.

Gamarra, G., Wong, F. J., Rivera, T. A. y Pujay, O. E. (2015). Estadística e investigación con aplicaciones de SPSS. Lima: San Marcos.

Gaona, I. (2013). "El bilingüismo guaraní castellano y su incidencia en la producción escrita de los alumnos al final del primer ciclo de la EEB". L a Plata, Argentina.

Hernández, R., Fernández, C. \& Baptista, P. (2010). Metodología de la investigación. $\left(5^{\circ}\right.$ ed.) México: McGraw-hill.

Julca, F. (2000). "Uso de las lenguas quechua y castellano en la escuela urbana": un estudio de caso. Cochabamba: (3ra ed.).

La Unesco, (1953). Empleo de las lenguas vernáculas en la enseñanza. París: Autor.
Lugo, A. (2007). Comprensión y producción de textos científicos. Ed. Fondo de publicación

Mayor, J. y otros. (2001). Estrategias metacognitivas. Aprender a Aprender y Aprender a Pensar. Ed. Trillas. México

Ministerio de Educación (2009). La lectura en PISA 2009. Marcos y pruebas de la evaluación. Madrid. Ministerio de Educación.

Morales, F. y otros (2009). Bilingüismo en el rendimiento académico de alumnos del Nivel Secundario de Instituciones Educativas Públicas en la Región de Ayacucho. Ayacucho: Instituto de Investigación de la Facultad de Ciencias de la Educación-UNSCH.

Moreno, F. (1998). Principios de sociolingüística y sociología del lenguaje. Barcelona: Ariel S.A.

Oré, R. (2012)Comprensión lectora, hábitos de estudio y rendimiento académico en estudiantes de primer año de una universidad privada de Lima Metropolitana. Lima-Perú

Padrón, E. (2001). Influencia de las estrategias de aprendizaje en la comprensión lectora del Inglés con propósitos específicos. Tesis de grado. Universidad del Zulia: Maracaibo, Venezuela

Pérez, H. (2006). Comprensión y producción de textos educativos. Ed. Cooperativa editorial magisterio

Pinzas, J. (2006). Guía de estrategias metacognitivas de comprensión lectora. Ministerio de Educación.

Real Academia Española, Diccionario de la lengua española, 23. ${ }^{\mathrm{a}}$ ed. Madrid: Espasa, 2014.

Ruíz, R. (2011). Lectura intensiva a la extensiva en la clase fundamentos didácticos. Granada.

Salas, P. (2012) EL desarrollo de la comprensión lectora en losestudiantes del tercer semestre 
del nivel mediosuperior de la Universidad Autónoma De Nuevo León.México

Smith, F. (2005). Comprensión de la lectura. Análisis psicolingüístico de la lectura y su aprendizaje. Ed. TrillasMéxico

Solé, I. (2000). Estrategias de lectura. Ed. Barcelona-España: Grao.

Valenzuela, C. (2007). Enseñanza del lenguaje, un nuevo enfoque. Edición 2012.Editorial: piedra santa. Guatemala.

Yucra, V. (2015). Bilingüismo $y$ producción de textos en estudiantes de secundaria. Ayacucho, 2015. Ayacucho: Posgrado de la Universidad César Vallejo.

\section{REVISTAS}

Acuña, E. y Eyzaguirre, N. (2012). Comprensión lectora de los estudiantes bilingües interculturales en el Perú. Aportes y desafíos a la propuesta pedagógica de EIB. Congreso lberoamericano de las Lenguas en la Educación y en la Cultura. Salamanca-España. Encontrado en: www.oei. Es/histórico/congresolenguas/comuni caciones.

Coronado, B y Ruiz, D (2010). Estrategias para la comprensión inferencial en la lectura del hipertexto telemático. Revista de la Universidad de Los Andes de Venezuela. Encontrado en: www.saber.ula.ve/bitstream/123456789/ 33600/1/articulo1.pdf

Fumero, F. (2009). Estrategias didácticas para la comprensión de textos. Una propuesta de investigación acción participativa en el aula. Revista de la Investigación y Posgrado UPEL. Encontrado en: revistas. upel.edu.ve/index.php/revinpost /article/view/909

Sánchez, F. (2006). La comprensión lectora en la escuela primaria.
Encontrado en dgfcms.sep.gob.mx/htm//Materiales/PRI/ Docs/PB07/Comp_lect_esc_prim.pd

Sotoca, E. (2013) La repercusión del bilingüismo en el rendimiento académico en alumnos de colegios públicos de la comunidad de Madrid. Revista Complutense de Educación. Encontrado en: https://revistas.ucm.es/index.php/RCED/ article/ download/ 41732/42946 